\title{
Study on Magnetized RF Discharge with Very Small-Diameter ${ }^{*)}$
}

\author{
Toshiki NAKAGAWA, Yoshitake SATO, Eiko TANAKA, Hiraku IWAYA, \\ Daisuke KUWAHARA and Shunjiro SHINOHARA \\ Tokyo University of Agriculture and Technology, 2-24-16 Naka-Cho, Koganei, Tokyo 184-8588, Japan
}

(Received 1 December 2014 / Accepted 17 February 2015)

\begin{abstract}
To investigate characteristics and estimate propulsion performance of a high-density radio frequency plasma with a very small diameter, we have developed the Small Helicon Device (SHD) and measured an electron density $n_{\mathrm{e}}$, an ion velocity $v_{\mathrm{i}}$ and an emission intensity of Ar II under various conditions such as a discharge diameter (down to $3 \mathrm{~mm}$ ), a mass flow rate and a gas species. Using quartz discharge tubes with inner diameter (i.d.) of 3, $10,20 \mathrm{~mm}$ with an Ar gas, $n_{\mathrm{e}}$ of $(2.5-6) \times 10^{12}$ (estimated from optical measurement), $\sim 2.5 \times 10^{12}$ and $\sim 2.0 \times$ $10^{12} \mathrm{~cm}^{-3}$, respectively, at $-40 \mathrm{~mm}$ downstream from an excitation antenna was obtained. Using the $20 \mathrm{~mm}$ i.d. tube with a $\mathrm{H}_{2}$ gas, $v_{\mathrm{i}} \sim 40 \mathrm{~km} / \mathrm{s}$ was achieved in the presence of the magnetic field gradient.
\end{abstract}

(C) 2015 The Japan Society of Plasma Science and Nuclear Fusion Research

Keywords: helicon plasma, small helicon device, electron density, emission intensity, ion velocity

DOI: $10.1585 /$ pfr. 10.3401037

\section{Introduction}

An electric propulsion system has a higher efficiency than a chemical one, and it suits for long term missions such as deep space explorations. However, a lifetime of the electric propulsion system, such as a Hall thruster, is limited by damage of electrodes contacting directly with a plasma. To solve this problem, we have proposed completely electrodeless helicon thruster using a high-density $\left(\sim 10^{13} \mathrm{~cm}^{-3}\right)$ helicon plasma [1-4]. In the case of producing the helicon plasma with a very small diameter, this source will contribute to the development of a lightweight and compact thruster such as an attitude control thruster, and that of a neutralizer. Here, simple thrusters can be seen in refs. [5-9]. The high-density, small plasma source may also have industrial applications, such as a coating of the inner wall of a thin tube.

In this paper, using the Small Helicon Device (SHD) $[10,11]$, we measured an electron density $n_{\mathrm{e}}$ and an ion velocity $v_{\mathrm{i}}$ using electrostatic probes to characterize smalldiameter helicon plasmas. However, probes may disturb the plasma flow, therefore sometimes it is not suitable for measurements in very small diameter. Instead, optical measurements are useful. We measured an emission intensity of $\mathrm{Ar}$ II $\left(I_{\mathrm{Ar} \text { II }}\right)$ with three small tubes, using a spectrometer to estimate $n_{\mathrm{e}}$ and compared this from a probe measurement. Here, if we can assume a Maxwellian electron energy distribution with a uniform electron temperature $T_{\mathrm{e}}$, and the non-saturated phase of the ionizing plasma, which holds good in our experimental conditions, $I_{\text {Ar II }}$ is proportional to $n_{\mathrm{e}}^{2}$, and $\sqrt{I_{\mathrm{Ar} \mathrm{II}}} / n_{\mathrm{e}}$ should be constant [12].

Furthermore, to estimate propulsion performance of a

author'se-mail: yukasenzai@gmail.com

*) This article is based on the presentation at the 24th International Toki Conference (ITC24). small-diameter plasma, we measured $n_{\mathrm{e}}$ and ion velocity $v_{\mathrm{i}}$ (relating to a thrust and a specific impulse) using a $20 \mathrm{~mm}$ diameter tube with different gas species of $\mathrm{He}$ and $\mathrm{H}_{2}$ in addition to Ar. Here, we can expect higher ion velocity with the lighter ions if the axial potential drop is the same.

\section{Experimental Setup}

Figure 1 shows a photograph and a schematic drawing of SHD. A small-diameter, quartz discharge tube was connected to a vacuum chamber (SUS316) in inner diameter of $165 \mathrm{~mm}$ and an axial length of $865 \mathrm{~mm}$, pumped by a rotary pump (CVD-050A, ULVAC) through a turbomolecular pump (TG200, Osaka Vacuum). Here, various diameter discharge tubes can be connected to the vacuum gauge port adapters [left sides of Figs. 1 (a) and (b)]. Pirani gauge and ion one were installed at the head of the discharge tube and also at the end of the chamber. The base pressure in the vacuum chamber was $\sim 7 \times 10^{-5} \mathrm{~Pa}$. Quartz

(a)

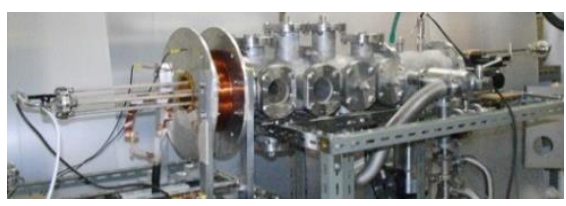

(b)

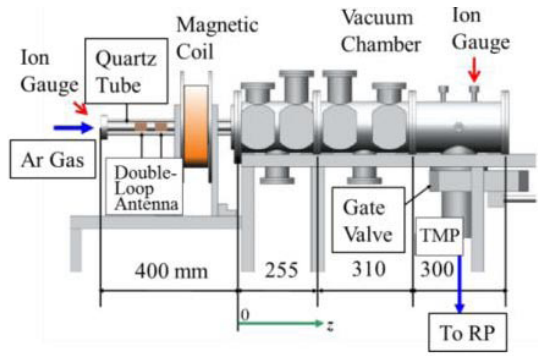

Fig. 1 (a) Photograph and (b) schematic drawing of SHD. 
tubes have inner diameters (i.d.s) of 3,10 and $20 \mathrm{~mm}$ with axial lengths of $453 \mathrm{~mm}$. The radio frequency (rf) antenna was a double-loop type at $z=-170 \sim-230 \mathrm{~mm}$, where $z$ $=0$ is defined in Fig. 1 (b), made of copper plates with a thickness of $0.2 \mathrm{~mm}$ and a width of $40 \mathrm{~mm}$.

An rf power supply (T145-6326CK, THAMWAY) can deliver $\mathrm{rf}$ power up to $2 \mathrm{~kW}$ with an $\mathrm{rf}$ frequency of 0.3 - 13.56 MHz, through a matching box (T020-6326AK, THAMWAY). A directional coupler in the matching box was connected to an oscilloscope (TDS2024B, Tektronix) to measure rf power $P_{\text {rf }}$ (subtracting the reflecting power from the incident one). Gases can be fed by two mass flow controllers (SEC-400MK3, HORIBASTEC: 0 - 30 sccm, $0.1 \mathrm{sccm}$ step, and SEC-Z512KX, HORIBASTEC: $0-1$ sccm, $0.01 \mathrm{sccm}$ ). To accelerate plasmas with the magnetic field gradient and stabilize them with a better confinement, a handmade solenoid coil $(90 \mathrm{~mm}$ i.d., $360 \mathrm{~mm}$ outer diameter and $120 \mathrm{~mm}$ axial length with 400 turns of winding coils) was used; the coil can produce the axial magnetic field of $28 \mathrm{G} / \mathrm{A}$ in the coil region and the typical operating coil current $I$ is $20 \mathrm{~A}$. To have reproducible discharges in the case of i.d. of $3 \mathrm{~mm}$ tube, a hot filament was installed in the vacuum chamber.

For the measurement of $n_{\mathrm{e}}, T_{\mathrm{e}}$ and $v_{\mathrm{i}}$, a single probe, a double probe and a Mach probe with a data logger (MEMORY HiCORDER 8855, $20 \mathrm{MS} / \mathrm{s}$, HIOKI) were used. Plasma emission intensities were measured by a CCD spectrometer (HR2000+, Ocean Optics, wavelength range: $360-792 \mathrm{~nm}$, resolution: $0.45 \mathrm{~nm}$ ). We chose $434.94 \mathrm{~nm}$ of the wavelength of Ar II. As was mentioned, $n_{\mathrm{e}}$ can be estimated, since it is proportional to $\sqrt{I_{\mathrm{Ar} I I}}$. The electron temperature, which is also needed to estimate $n_{\mathrm{e}}$ and $v_{\mathrm{i}}$, was assumed to be $5 \mathrm{eV}$ (typical value) in those measurements.

\section{Experimental Results}

Figures 2 and 3 show $n_{\mathrm{e}}$ and $I_{\mathrm{Ar} \text { II }}$ at $z=-60 \mathrm{~mm}$ (in the quartz tube and downstream of the rf antenna), respectively, as a function of $P_{\mathrm{rf}}$ with an i.d. of $20 \mathrm{~mm}$ tube with the Ar gas (the product of the effective solid angle and path length is $L=0.959$ str.mm). A solenoid coil was placed at $z=-155 \mathrm{~mm}$, to produce the magnetic field. Although $n_{\mathrm{e}}$ did not reach $10^{13} \mathrm{~cm}^{-3}, n_{\mathrm{e}}$ and $I_{\text {Ar II }}$ increased with $P_{\text {rf }}$ under all Flow Rate $F R$ and magnetic coil current $I$. In the case of $F R$ of $20 \mathrm{sccm}$ without the magnetic field, $n_{\mathrm{e}}$ jump by a factor of $\sim 20$ was observed at $P_{\mathrm{rf}} \sim 200 \mathrm{~W}$, and $I_{\mathrm{Ar} \text { II }}$ was too weak to be observed before the density jump. The jump is considered to be a mode transition from a Capacitively Coupled Plasma (CCP) to an Inductively Coupled Plasma (ICP). $I_{\mathrm{Ar} \text { II }}$ showed the opposite dependence on the magnetic field.

Figures 4 and 5 show $T_{\mathrm{e}}$ by a double probe and the ratio of the square root of $I_{\mathrm{Ar} \text { II }}$ to $n_{\mathrm{e}}$ as a function of $P_{\mathrm{rf}}$ respectively. $T_{\mathrm{e}}$ decreased slightly with $P_{\mathrm{rf}}$ especially without the magnetic field. Since $I_{\mathrm{Ar} \text { II }}$ is proportional to $n_{\mathrm{e}}^{2}$,

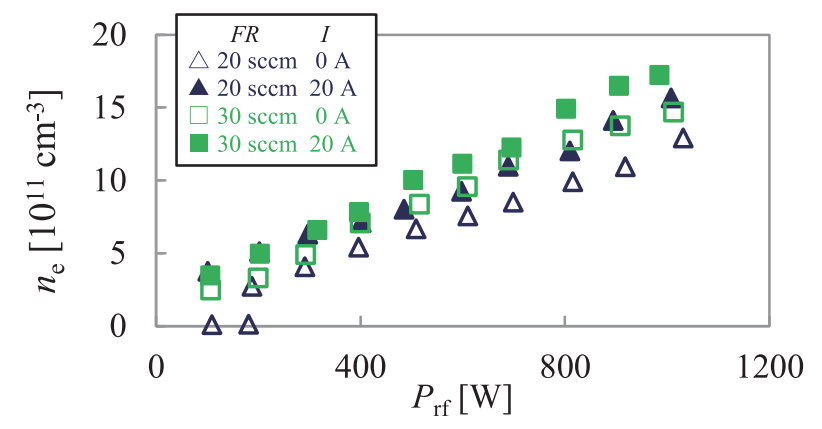

Fig. $2 n_{\mathrm{e}}$ vs. $P_{\text {rf }}$ with a $20 \mathrm{~mm}$ i.d. tube, changing $F R$ and $I(z$ $=-60 \mathrm{~mm}$ ).

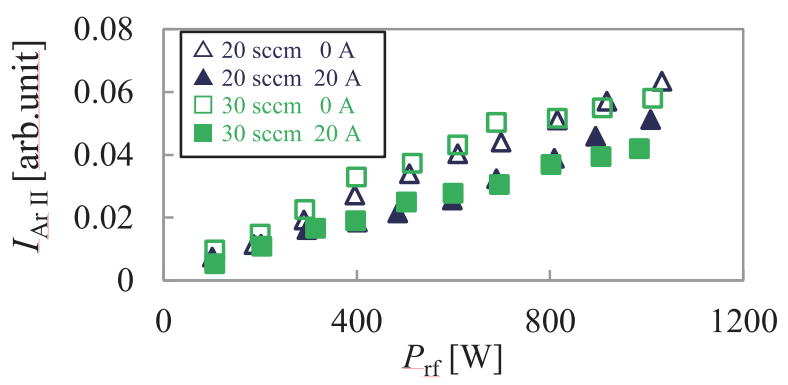

Fig. $3 I_{\text {Ar II }}$ vs. $P_{\text {rf }}$ with a $20 \mathrm{~mm}$ i.d. tube, changing $F R$ and $I(z$ $=-60 \mathrm{~mm}$ ).

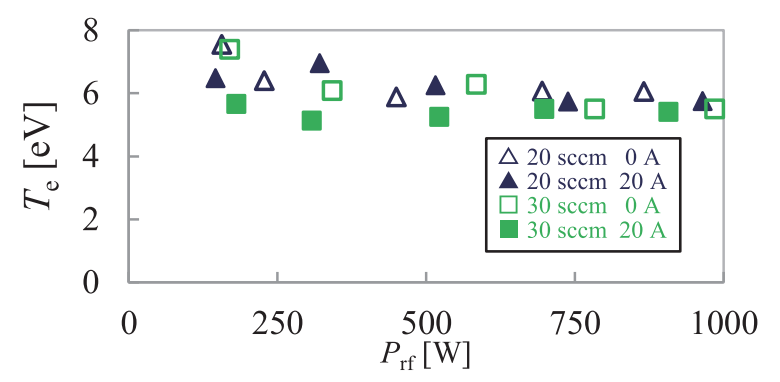

Fig. $4 T_{\mathrm{e}}$ vs. $P_{\mathrm{rf}}$ with a $20 \mathrm{~mm}$ i.d. tube, changing $F R$ and $I(z$ $=-60 \mathrm{~mm}$ ).

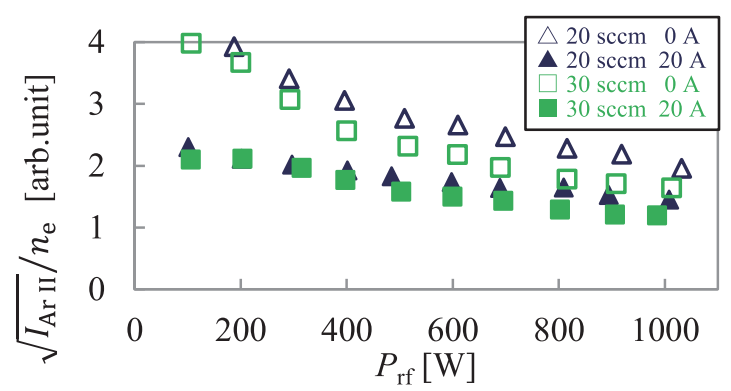

Fig. $5 \sqrt{I_{\mathrm{Ar} \mathrm{II}}} / n_{\mathrm{e}}$ vs. $P_{\mathrm{rf}}$ with a $20 \mathrm{~mm}$ i.d. tube, changing $F R$ and $I(z=-60 \mathrm{~mm})$.

$\sqrt{I_{\mathrm{Ar} \mathrm{II}}} / n_{\mathrm{e}}$ should be constant if uniform $T_{\mathrm{e}}$ is assumed, as was mentioned. However, $\sqrt{I_{\mathrm{Ar} \mathrm{II}}} / n_{\mathrm{e}}$ decreased with $P_{\mathrm{rf}}$ and larger in cases of without the magnetic field under low rf powers. This may be due to the fact that $I_{\mathrm{Ar}}$ II is proportional to the cross section, which increases strongly with $T_{\mathrm{e}}$ [13]. 


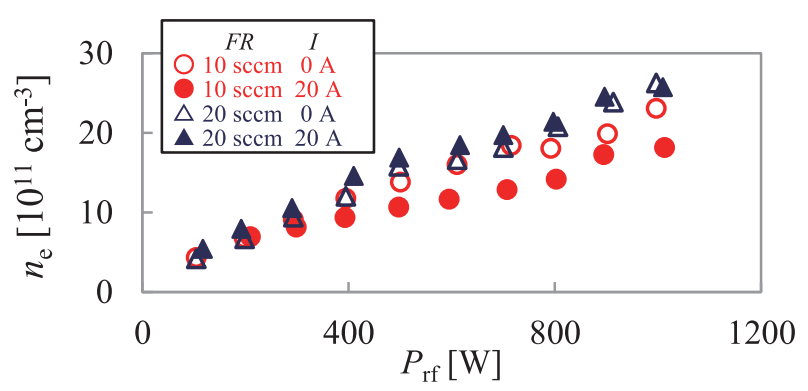

Fig. $6 n_{\mathrm{e}}$ vs. $P_{\mathrm{rf}}$ with a $10 \mathrm{~mm}$ i.d. tube, changing $F R$ and $I(z$ $=-60 \mathrm{~mm})$.

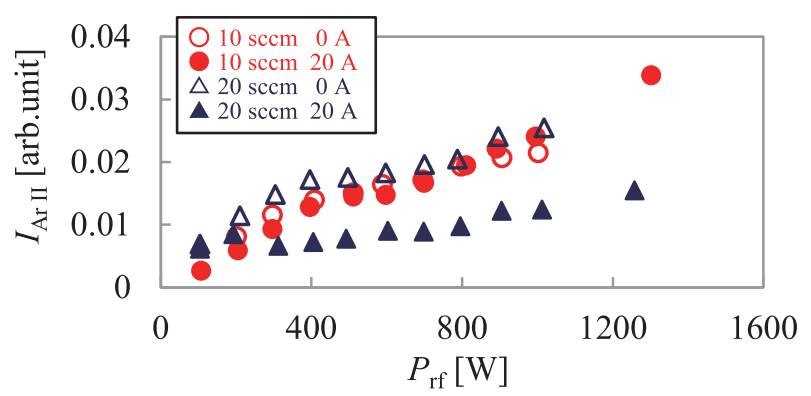

Fig. $7 I_{\mathrm{Ar} \text { II }}$ vs. $P_{\mathrm{rf}}$ with a $10 \mathrm{~mm}$ i.d. tube, changing $F R$ and $I(z$ $=-60 \mathrm{~mm})$.

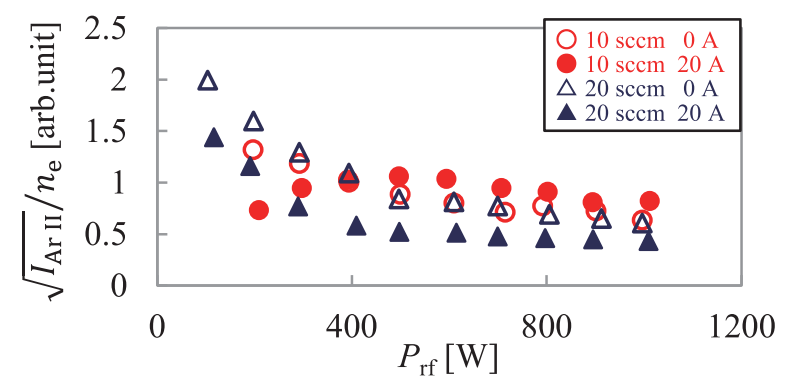

Fig. $8 \sqrt{I_{\mathrm{Ar} \text { II }}} / n_{\mathrm{e}}$ vs. $P_{\mathrm{rf}}$ with a $10 \mathrm{~mm}$ i.d. tube, changing $F R$ and $I(z=-60 \mathrm{~mm})$.

Figures 6 and 7 show $n_{\mathrm{e}}$ and $I_{\mathrm{Ar} \text { II }}$ at $z=-60 \mathrm{~mm}$ as a function of $P_{\text {rf }}$ with i.d. of $10 \mathrm{~mm}$ tube using an Ar gas, respectively ( $L: 0.452$ str.mm). A solenoid coil was placed at $z=-155 \mathrm{~mm}$. Although this shows larger $n_{\mathrm{e}}$ with $10 \mathrm{~mm}$ i.d. than that of $20 \mathrm{~mm}$ i.d. case, this density range can be expected from a plasma production efficiency [14]. $I_{\mathrm{Ar}}$ II is lower due to a lower receiving volume, and the density jump was not observed. In the case of $F R$ of $10 \mathrm{sccm}$ and $I=20 \mathrm{~A}, n_{\mathrm{e}}$ was the lowest and $\sqrt{I_{\mathrm{Ar} I \mathrm{II}}} / n_{\mathrm{e}}$ (shown in Figure 8) also decreased with $P_{\text {rf }}$. This value of $\sqrt{I_{\mathrm{Ar} \mathrm{II}}} / n_{\mathrm{e}}$ is consistent with that in the case of $20 \mathrm{~mm}$ i.d., considering also $L$ using a fiberscope of the monochromator.

Figure 9 shows $I_{\mathrm{Ar} \text { II }}$ at $z=-60 \mathrm{~mm}$ as a function of $P_{\mathrm{rf}}$ with i.d. of $3 \mathrm{~mm}$ using with an $\mathrm{Ar}$ gas $(L$ : 0.072 str.mm). A solenoid coil was placed at $z=-155 \mathrm{~mm}$. Although we have succeeded in rf plasma discharges, $n_{\mathrm{e}}$ could not be measured due to the comparable size of the probe to the quartz tube. The discharge was unstable and plasmas could not be produced at $P_{\mathrm{rf}}<700 \mathrm{~W}$. The plasma

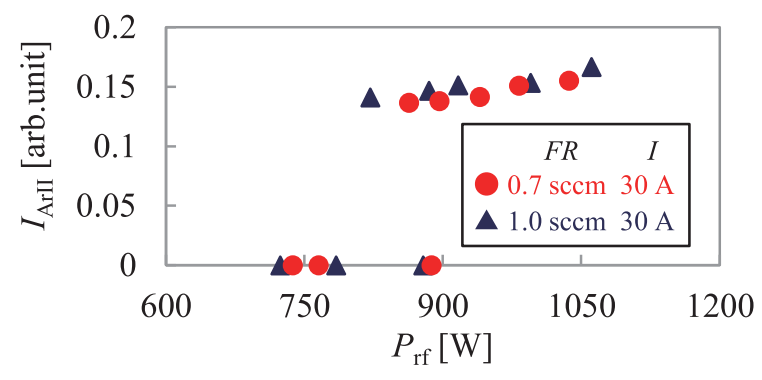

Fig. $9 I_{\mathrm{Ar} \text { II }}(z=-60 \mathrm{~mm})$ vs. $P_{\mathrm{rf}}$ for $3 \mathrm{~mm}$ i.d. tube changing $F R$.

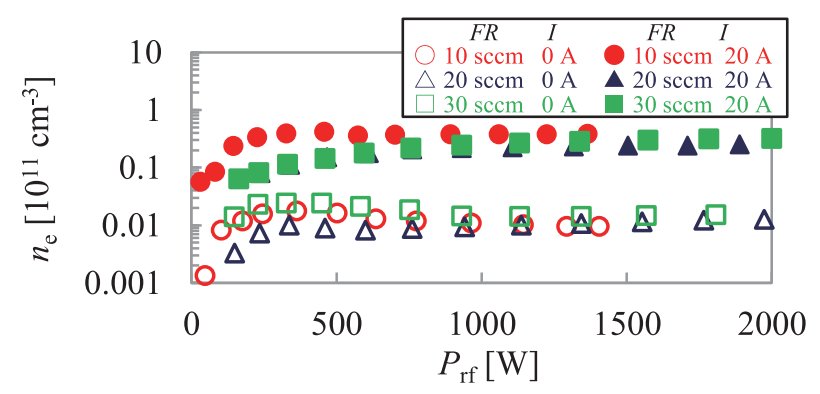

Fig. $10 n_{\mathrm{e}}$ vs. $P_{\text {rf }}$ with a He gas, changing $F R$ and $I(z=-40 \mathrm{~mm})$.

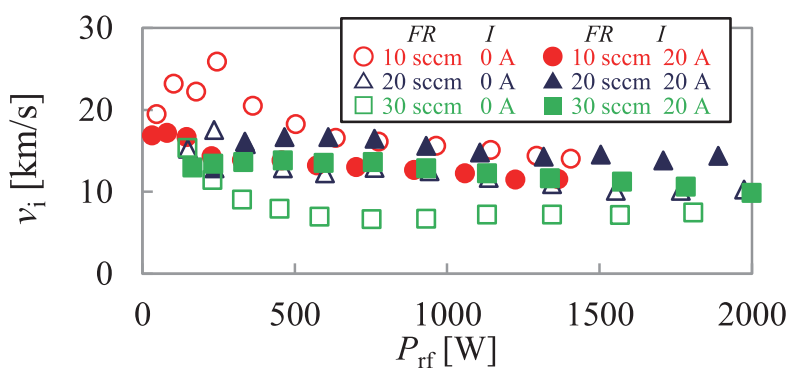

Fig. $11 v_{\mathrm{i}}$ vs. $P_{\mathrm{rf}}$ with He gas, changing $F R$ and $I(z=-40 \mathrm{~mm})$.

plume was localized and did not reach the optical measurement point of $z=-60 \mathrm{~mm}$ in the lower $P_{\mathrm{rf}}$ region. Here, $I_{\text {Ar II }} \sim 0.15$ for $P_{\text {rf }} \sim 1,000 \mathrm{~W}$ was obtained at 0.7 and 1.0 sccm. From the results with 10 and $20 \mathrm{~mm}$ i.d., considering $L$, we can estimate that $n_{\mathrm{e}}$ for i.d. of $3 \mathrm{~mm}$ was $(2.5-6)$ $\times 10^{12} \mathrm{~cm}^{-3}$.

Next, we will show the plasma performance including the ion velocity, changing gas species. A solenoid coil was placed at $z=-90 \mathrm{~mm}$. Figures 10 and 11 show $n_{\mathrm{e}}$ and $v_{\mathrm{i}}$ at $z=-40 \mathrm{~mm}$ (in the quartz tube region and away from the antenna region, having the large magnetic field gradient) as a function of $P_{\mathrm{rf}}$ with i.d. of $20 \mathrm{~mm}$ tube using a He gas, respectively. Here, for a Mach probe measurement, an unmagnetized model was used (model constant $\kappa=1.26)[15,16]$. In the experiment using the Ar gas, $n_{\mathrm{e}}$ of $\sim 3 \times 10^{12} \mathrm{~cm}^{-3}$ and $v_{\mathrm{i}}$ of $\sim 1.5 \mathrm{~km} / \mathrm{s}$ was obtained for $P_{\text {rf }} \sim 1,500 \mathrm{~W}$ with and without magnetic fields, respectively. In He discharges, $n_{\mathrm{e}} \sim 3 \times 10^{10}$ and $10^{9} \mathrm{~cm}^{-3}$ was obtained for $P_{\mathrm{rf}} \sim 1,500 \mathrm{~W}$ with and without the magnetic fields, respectively. Here, $n_{\mathrm{e}}$ was lower than the case of Ar, and plasma emission intensities were weaker, because the 


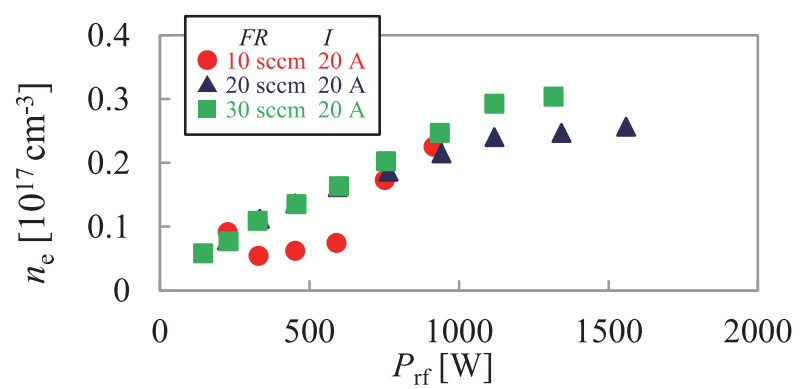

Fig. $12 n_{\mathrm{e}}$ vs. $P_{\mathrm{rf}}$ with a $\mathrm{H}_{2}$ gas, changing $F R$ and $I(z=-40 \mathrm{~mm})$.

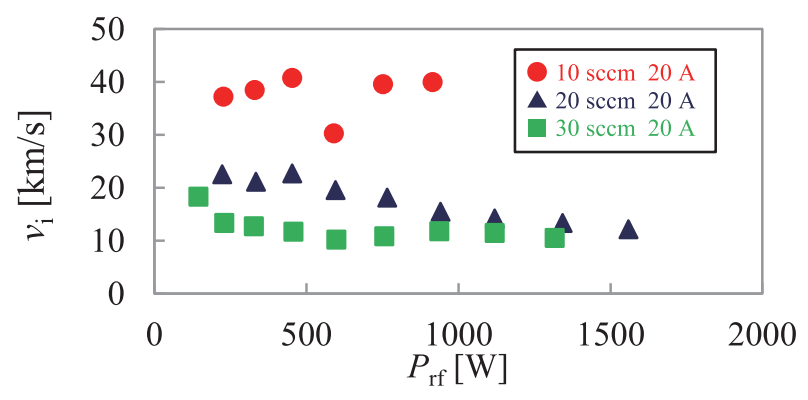

Fig. $13 v_{\mathrm{i}}$ vs. $P_{\text {rf }}$ with a $\mathrm{H}_{2}$ gas, changing $F R$ and $I(z=-40 \mathrm{~mm})$.

first ionization energy of He is higher than Ar. Since the molecular weight of $\mathrm{He}$ is about one tenth of that of $\mathrm{Ar}, v_{\mathrm{i}}$ of He is much higher than Ar; with $P_{\mathrm{rf}} \sim 1,500 \mathrm{~W}, v_{\mathrm{i}} \sim 15$ $\mathrm{km} / \mathrm{s}$ was obtained (typical ion Mach number $M_{\mathrm{i}} \sim 1.5$ ) and saturated at $P_{\mathrm{rf}}>200 \mathrm{~W}$ under all conditions.

Figures 12 and 13 show $n_{\mathrm{e}}$ and $v_{\mathrm{i}}$ at $z=-40 \mathrm{~mm}$ as a function of $P_{\mathrm{rf}}$ with i.d. of $20 \mathrm{~mm}$ with a $\mathrm{H}_{2}$ gas, respectively. Without the magnetic field $(I=0), n_{\mathrm{e}}$ was low and discharge was unstable, therefore we could not measure plasma parameters in the quartz tube region. Although the first ionization energy of $\mathrm{H}_{2}$ is lower than that of Ar, $n_{\mathrm{e}}$ of $\mathrm{H}_{2}$ was lower than that of Ar because $\mathrm{H}_{2}$ is a diatomic molecule [17]. With $F R$ of $20 \mathrm{sccm}$ and $I$ of $20 \mathrm{~A}$, $v_{\mathrm{i}} \sim 40 \mathrm{~km} / \mathrm{s}\left(M_{\mathrm{i}} \sim 1.8\right)$ was obtained, and it is the highest velocity, leading to the specific impulse of $\sim 4,000 \mathrm{~s}$ for protons. In the cases of $\mathrm{Ar}$ and $\mathrm{He}, v_{\mathrm{i}}$ did not change so much for all the conditions $(\sim 1.5 \mathrm{~km} / \mathrm{s}$ with an Ar ion and $\sim 12 \mathrm{~km} / \mathrm{s}$ with a He ion). However, in the case of $\mathrm{H}_{2}, v_{\mathrm{i}}$ increased at lower $F R$, which is considered as a reduction of collision frequency due to a low pressure.

\section{Concluding Remarks}

We have obtained the following results with very small diameters in magnetized rf discharges.

1. High-density plasmas were produced with small diameter discharge tubes; i.d. of 3, 10 and $20 \mathrm{~mm}$ with an Ar gas. By the Langmuir probe and the spectrometer, $n_{\mathrm{e}}$ and $I_{\mathrm{Ar} \text { II }}$ were measured. Here, $T_{\mathrm{e}}$ was also measured and showed a slight decrease with $P_{\mathrm{rf}}$.

2. High-density plasmas with high flow velocities were measured by a Mach probe in the case of i.d. of $20 \mathrm{~mm}$ using $\mathrm{He}$ and $\mathrm{H}_{2}$ gases in addition to Ar one. Here, $n_{\mathrm{e}}$ of $\sim 3 \times 10^{10} \mathrm{~cm}^{-3}$ and $v_{\mathrm{i}}$ of $\sim 40 \mathrm{~km} / \mathrm{s}$ was obtained using a $\mathrm{H}_{2}$ gas at $F R=10 \mathrm{sccm}, I=20 \mathrm{~A}$ and $P_{\mathrm{rf}} \sim 1,000 \mathrm{~W}$.

3. In the cases of i.d.s of 10 and $20 \mathrm{~mm}, \sqrt{I_{\mathrm{Ar} \text { II }}} / n_{\mathrm{e}}$ was not constant and decreased with an increase of $P_{\mathrm{rf}}$, which can be considered that $T_{\mathrm{e}}$ was lower in the higher $P_{\text {rf }}$ region than the lower one, ascertained by a probe measurement. In the case of i.d. $3 \mathrm{~mm}$, where a probe measurement is impossible, $n_{\mathrm{e}}$ was estimated to be $(2.5-6) \times 10^{12} \mathrm{~cm}^{-3}$ from an optical measurement.

As for a thrust performance, a rough estimate shows that the total thrusts $F_{\text {total }}$ ( $=$ ion thrust $F_{\mathrm{i}}+$ neutral thrust $F_{0}$ ) are on the order of $\sim 100,10$ and $10 \mu \mathrm{N}$ for Ar, He and $\mathrm{H}_{2}$ gases, respectively. Although ion velocity is high, effective specific impulse $I_{\mathrm{sp}}\left(=F_{\text {total }} / \dot{m}_{0} g\right.$, where $\dot{m}_{0}$ : neutral mass flow rate, $g$ : acceleration of gravity) is low $(<100 \mathrm{~s})$ due to a high neutral density (low ionization degree).

In this experiment, we have measured $n_{\mathrm{e}}$ and $v_{\mathrm{i}}$ with small diameters. To measure them with no perturbation to very small diameter plasmas, we are planning to use a fine resolution spectrometer (focal length $1.5 \mathrm{~m}$, minimum resolution $\sim 0.005 \mathrm{~nm}$ ). In addition, to measure $n_{\mathrm{e}}$ and $T_{\mathrm{e}}$, we are developing a code based on Collisional Radiative (CR) model, calculating line intensity ratios using Ar I lines, and estimate $n_{\mathrm{e}}$ and $T_{\mathrm{e}}[12,18]$.

[1] R.W. Boswell, Phys. Lett. 33A, 457 (1970).

[2] S. Shinohara, Jpn. J. Appl. Phys. 36, 4695 (1997).

[3] R.W. Boswell and F.F. Chen, IEEE Trans. Plasma Sci. 25, 1229 (1997).

[4] F.F. Chen and R.W. Boswell, IEEE Trans. Plasma Sci. 25, 1245 (1997).

[5] C. Charles, J. Phys. D, Appl. Phys. 42, 18 (2009).

[6] E. Ahedo and M. Merino, Phys. Plasmas 17, 073501 (2010).

[7] K. Takahashi, C. Charles, R.W. Boswell and T. Fujiwara, Phys. Rev. Lett. 107, 35002 (2011).

[8] S. Shinohara et al., IEEE Trans. Plasma Sci. 42, 1245 (2014).

[9] A. Fruchtman, Phys. Rev. Lett. 96, 065002 (2006).

[10] D. Kuwahara, A. Mishio, T. Nakagawa and S. Shinohara, Rev. Sci. Instrum. 84, 103502 (2013).

[11] T. Nakagawa, S. Shinohara, D. Kuwahara, A. Mishio and H. Fujitsuka, JPS Conf. Ser. 1, 1245 (2014).

[12] S. Waseda, H. Fujitsuka, S. Shinohara, D. Kuwahara, M. Sakata and H. Akatsuka, Plasma Fusion Res. 9, 3406125 (2014).

[13] T. Shoji, Private communication.

[14] S. Shinohara et al., Phys. Plasmas 16, 057104 (2009).

[15] M. Hudis and L.M. Lidsky, J. Appl. Phys. 41, 5011 (1970).

[16] K.S. Chung, I.H. Hutchinson, B. Labombard and R.W. Boswell, Phys. Fluids B1, 2229 (1989).

[17] H. Kikuchi, Y. Hukui, Y. Sakawa and T. Shoji, Phys. Plasmas 10, 521 (2003).

[18] J. Vlček, J. Phys. D, Appl. Phys. 22, 623 (1989). 